\title{
Conventional Chemoembolization for Hepatocellular Carcinoma: Role of Cone-Beam Computed Tomography Guidance
}

\author{
In Joon Lee ${ }^{1}$, Jin Wook Chung ${ }^{2}$ \\ 'Department of Radiology, National Cancer Center, Goyang; ${ }^{2}$ Department of Radiology, Seoul National University Hospital, Seoul \\ National University College of Medicine, Seoul, Korea
}

Received Feb. 9, 2019

Revised Mar. 11, 2019

Accepted Mar. 12, 2019
Conventional chemoembolization using Lipiodol-based regimens was introduced in the 1980 s, and it is currently recommended as the primary treatment modality for patients with unresectable, intermediate, or locally advanced hepatocellular carcinoma (HCC) by the international guidelines. For better therapeutic efficacy and safety, chemoembolization should be performed as selectively as possible through tumor-feeding arteries, based on the detection of arterial supply to the HCC. With the technical advancement of flat-panel detector, cone-beam computed tomography (CBCT) is mounted on the C-arm of the angiographic machine. CBCT facilitates the detection of small occult HCCs and fine tumor-feeding arteries, recognition of extrahepatic collateral supply, navigation of a microcatheter to the target feeding arteries, prevention of non-target embolization, and intraprocedural assessment of the completeness of treatment with chemoembolization. These functions performed by CBCT ultimately improve the safety and efficacy of chemoembolization and may contribute to improving the prognosis of the patient with HCC. (J Liver Cancer 2019;19:19-29)

Keywords: Hepatocellular carcinoma; Therapeutics; Cone-beam computed tomography

\section{INTRODUCTION}

Conventional transarterial chemoembolization (cTACE) using Lipiodol-based regimens was introduced in the 1980s, and its therapeutic efficacy in unresectable hepatocellular carcinoma (HCC) was reinforced by two randomized controlled trials. ${ }^{1,2}$ Currently, chemoembolization is recommended for patients with HCC as a palliative treatment modality by international guidelines ${ }^{3-6}$ and is more widely used

\section{Corresponding author: Jin Wook Chung}

Department of Radiology, Seoul National University Hospital, Seoul National University College of Medicine, 101 Daehak-ro, Jongno-gu, Seoul 03080, Korea

Tel. +82-2-2072-2584, Fax. 82-2-743-6385

E-mail; chungjw@snu.ac.kr

https://orcid.org/0000-0002-1090-6872 than guideline recommendations in real-world practice. ${ }^{7}$ Therefore, it is very important to improve the outcome of cTACE. For better therapeutic efficacy and safety of cTACE, it should be performed as selectively as possible through the tumor-feeding arteries. ${ }^{8-10}$ With conventional angiographic systems, multiple angiography runs are usually needed to identify all the tumor-feeding arteries of the multiple and large tumors. In addition, it is not always easy to navigate a microcatheter to the target point through tortuous and branching arteries.

Recently, flat-panel detector systems have enabled us to perform real-time fluoroscopy, digital subtraction angiography (DSA), and cone-beam computed tomography (CBCT) in a single angiography unit. ${ }^{11,12}$ CBCT remarkably improved cTACE by providing high-resolution hepatic arteriographic 
images and three-dimensional (3D) roadmaps for selective catheterization, and it has become an essential tool for highly selective chemoembolization. ${ }^{13}$ In this article, the role of CBCT guidance in superselective chemoembolization with Lipiodol-based emulsion has been reviewed.

\section{WHY A SUPERSELECTIVE PROCEDURE?}

Because the majority of patients with HCC have underlying liver cirrhosis and impaired liver function, the overall survival is largely dependent on both tumor control and preservation of liver function. ${ }^{4}$ To maximize local tumor control and to minimize liver damage, chemoembolization should be performed as selectively as possible through the tumor-feeding arteries.

The intra-arterial treatment options for HCCs are based on the assumption that HCCs are almost exclusively supplied by hepatic arteries. However, exclusive hepatic arterial supply is only true in well-encapsulated, nodular HCCs. In multistep hepatocarcinogenesis of HCC, portal venous supply remains in the early stage. ${ }^{14}$ In advanced HCCs with a replacing or infiltrative growth pattern, the leading edge of the growing tumor receives dual blood supply from the hepatic artery and portal vein. In moderately differentiated HCCs, which have a pseudocapsule, peritumoral portal veins become tumor drainage vessels. Microsatellites may develop in the peritumoral area. ${ }^{15}$ The persistent portal venous supply or microsatellites can be one of the major causes of incomplete treatment of HCCs by chemoembolization.

Lipiodol-based emulsion is advantageous for chemoembolization because of its physical properties. ${ }^{16,17}$ It can pass through a fine tumor-feeding artery, reach the tumor vessels, penetrate deeply into the tumor capsule, and accumulate in the peritumoral portal veins through either presinusoidal arterioportal communication or the tumor drainage route. ${ }^{18}$ In a retrospective analysis of the clinical outcome of cTACE, local tumor recurrence was found to be significantly lower when more portal veins were visualized with Lipiodol during the procedure. When the peritumoral portal veins were extensively filled with Lipiodol, at least two-thirds of small HCCs were completely necrotized. ${ }^{19,20}$ However, it should be kept in mind that this ideal endpoint of "oily portogram" in cTACE can be achieved only in the case of selective catheterization of tumor-feeding arteries. Hence, a superselective procedure is essential to take advantage of Lipiodol-based emulsion in cTACE (Fig. 1).

\section{CBCT HEPATIC ARTERIOGRAPHY}

The flat-panel detector has better contrast and spatial resolution than image intensifier systems and provides CBCT images by obtaining whole volume data set in a single $\mathrm{C}$-arm rotation. Compared to multidetector CT (MDCT), CBCT has lower soft tissue contrast resolution because of lower detective quantum efficiency and lower tube voltage but higher spatial resolution because of a smaller focal spot and a larger matrix. ${ }^{21}$ Furthermore, hepatic arteries can be better depicted with CBCT because of the direct injection of contrast media from target vessels instead of the peripheral injection of MDCT scanning. ${ }^{22-24}$

Herein, we will outline the typical process of CBCT scanning that was followed by our institutions. ${ }^{25}$ Before CBCT scanning, angiography of the celiac trunk and superior mesenteric artery was performed using a 5-Fr catheter (RH; Cook, Bloomington, IN, USA) to evaluate the vascular anatomy and blood flow. The arms of the patients were positioned above the head to avoid streak artifacts from the forearms in CBCT hepatic arteriography. ${ }^{26}$ Rotational hepatic arteriography and CBCT scanning were performed at the common hepatic artery (or proper hepatic artery) using a 5-Fr catheter or a 2.8-Fr microcatheter (Cantata; Cook) with a continuous injection of contrast media during CBCT scanning at a flow rate of 2-4 mL/s after 4-6 seconds of X-ray delay, which was determined by considering the blood flow of the artery being injected, maximum flow rate of the catheters, and the total amount of contrast material needed. To avoid diaphragmatic motion artifact, a good breath-hold of the patient is important because it commonly takes 5-10 seconds to perform CBCT hepatic arteriography. In patients with hepatic artery variation (for example, the right hepatic artery replaced from the superior mesenteric artery), additional $\mathrm{CBCT}$ can be obtained at the right or left hepatic artery, as 

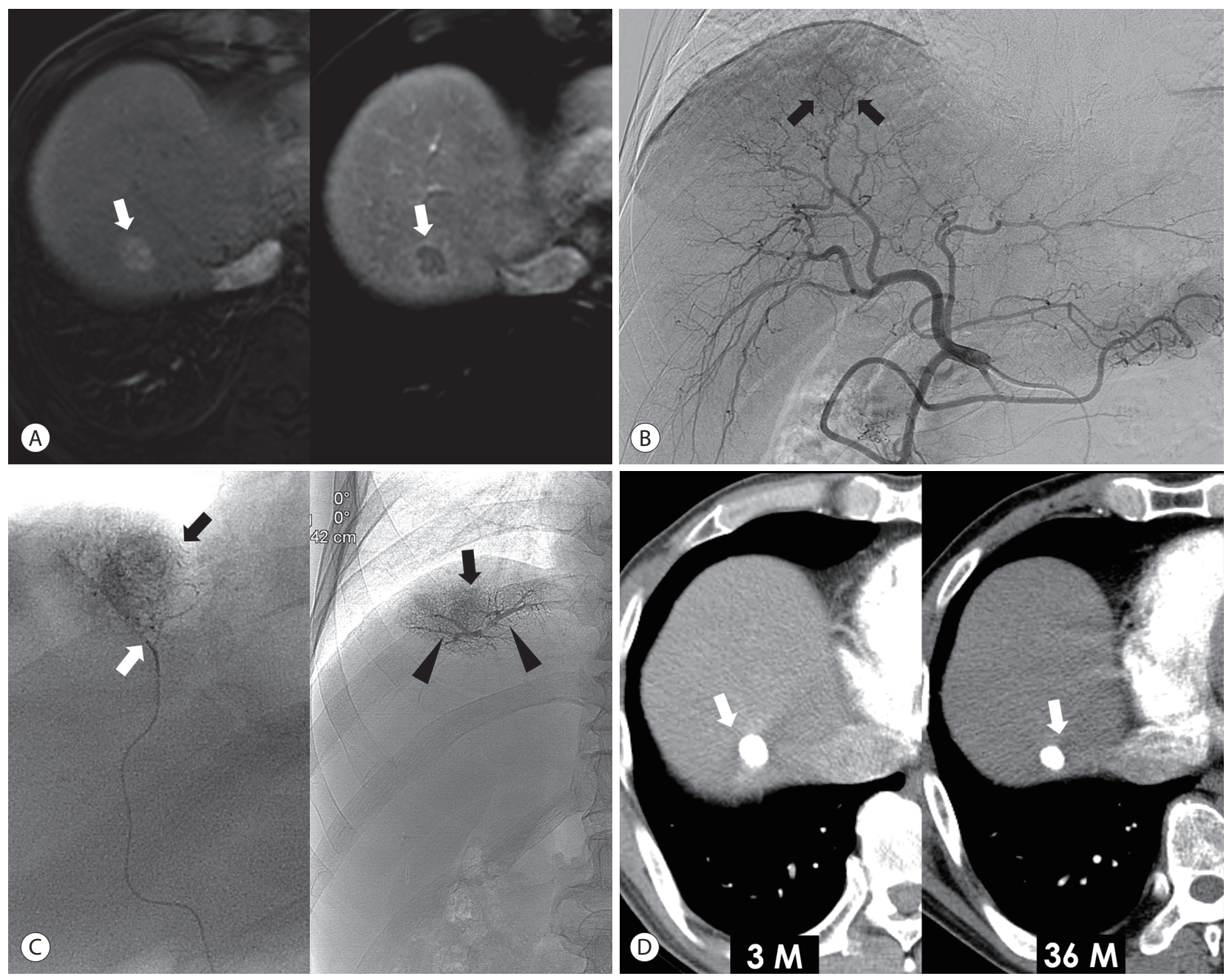

Figure 1. Superselective cTACE for a single small tumor in a 60-year-old man. (A) Liver MRI shows a $1.8 \mathrm{~cm}$ nodular tumor (arrows) with arterial enhancement (left side) and delayed washout (right side) in the segment 8 of the liver. (B) Common hepatic arteriography shows a faint enhancing tumor (arrows). (C) The tip (white arrow) of microcatheter is placed at the tumor-feeding artery, and the Lipiodol-based emulsion is infused. The portal veins (arrowheads) and tumor (black arrows) are visualized in the fluoroscopy image. (D) 3-month (left side) and 36-month (right side) follow-up CT shows dense Lipiodol accumulation at the tumor (arrows) without local recurrence. CTACE, conventional transarterial chemoembolization; MRI, magnetic resonance imaging; CT, computed tomography.

required. The parameters of CBCT vary with angiography systems and manufacturers. The rotational CBCT hepatic arteriography was performed during a single breath-hold of 6 seconds with a $0.5^{\circ}$ increment, $512 \times 512$ matrix in projections, total angle of $200^{\circ}$ at approximately $33^{\circ}$ per second, systemic dose of approximately 0.36 Gy per frame, and 419 projections, using angiography systems (AXIOM Artis Zee; Siemens, Erlangen, Germany). The acquired volume data set was transferred to the affiliated workstation for 3D image reconstruction, which included multiplanar reformation
(MPR), maximum intensity projection (MIP), and volume rendered images (Fig. 2).

Because the C-arm rotation time (5-10 seconds) was longer in CBCT than MDCT, breath-hold was important to obtain high-quality CBCT hepatic arteriograms suitable for superselective chemoembolization. In our previous study, the image quality of CBCT hepatic arteriography was good enough to trace subsegmental hepatic arteries in about 90\% of patients. ${ }^{25}$ Diaphragmatic motion was the most significant determinant of image quality, and CBCT could not be per- 

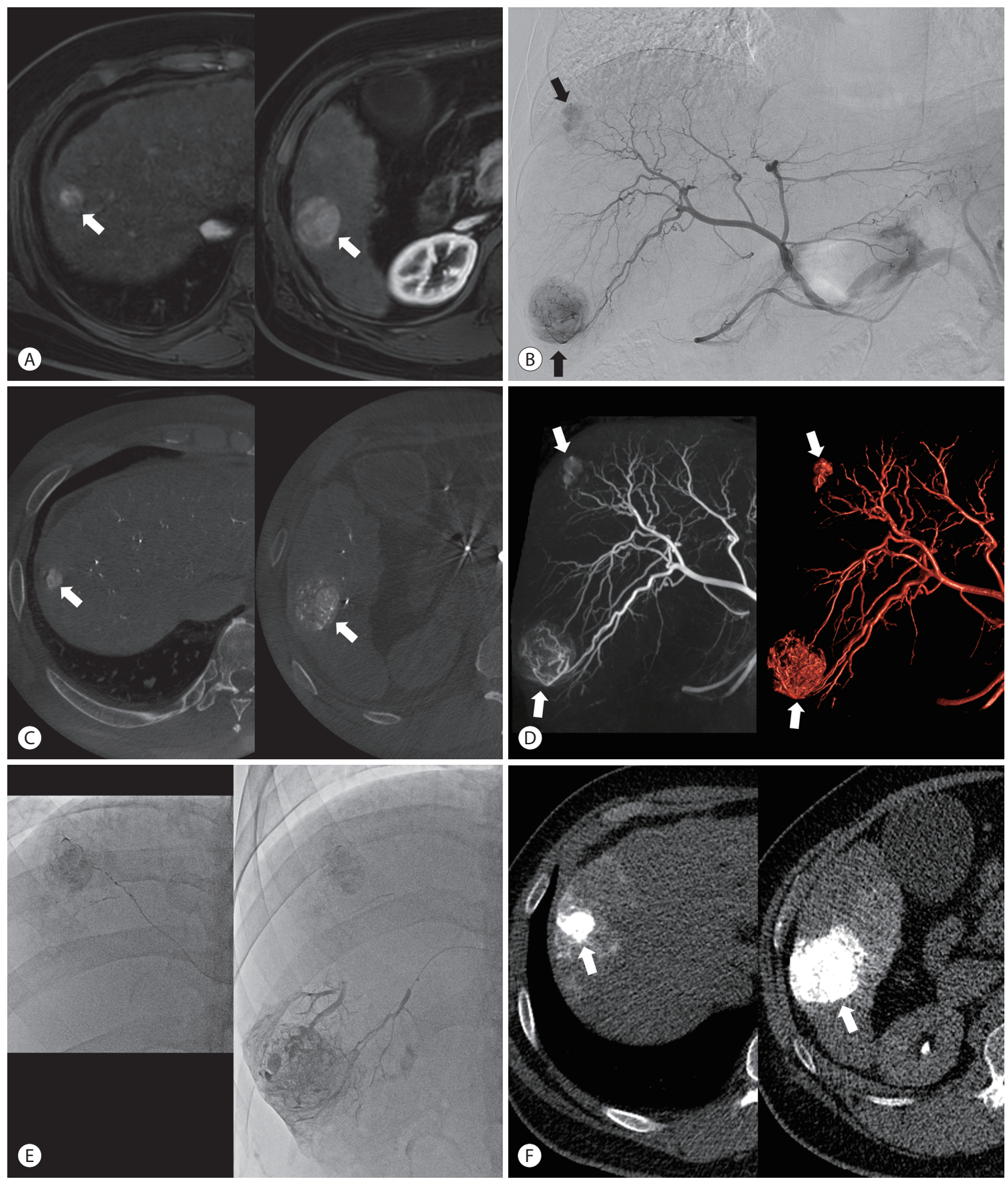

Figure 2. CBCT-guided CTACE for two nodular tumors in a 45-year-old man. (A) The arterial phase image of liver MRI shows two enhancing tumors (arrows) in segment 8 (left side) and segment 6 (right side). (B) Two enhancing tumors (arrows) are demonstrated in the common hepatic arteriography. (C) Axial image of CBCT shows two enhancing tumors (arrows) that are correlated with the preprocedural MRI. (D) The tumors (arrows) and their tumor-feeding arteries can be analyzed by using maximum intensity projection image (left side) and volume rendered image (right side). (E) Superselective chemoembolization is performed through each tumor-feeding artery. (F) Immediate, unenhanced CT shows dense Lipiodol accumulation in the tumors (arrows). CBCT, conebeam computed tomography; cTACE, conventional transarterial chemoembolization; MRI, magnetic resonance imaging; $\mathrm{CT}$, computed tomography. 
formed in 3\% of patients because of the long breath-hold requisite.

\section{ADVANTAGES OF CBCT GUIDANCE IN CHEMOEMBOLIZATION}

Most patients with HCCs have multiple tumors of variable size, vascularity, and feeders. For complete chemoembolization, it is important to depict small tumors with subtle arterial enhancement and with all the feeding arteries. Following are the advantages of CBCT guidance in superselective chemoembolization.

\section{Detection of small occult lesions}

HCCs have higher rates of multiplicity and recurrence due to either intrahepatic metastasis or multicentric de novo carcinogenesis. Therefore, sub-centimeter-sized metastatic satellite nodules or de novo lesions may not be visible with imaging studies at the initial presentation or during the followup. Some early-stage HCCs may not be visible in CT or magnetic resonance imaging (MRI) even with the steady increase of serum tumor markers.

Because CBCT shows high sensitivity in tumor detection, it is possible to detect the small occult HCCs that are not detected by CT or MRI. ${ }^{27-29}$ In our clinical practice, small HCC nodules (usually sub-centimeter in size) were additionally found by CBCT hepatic arteriography in 10-20\% of chemoembolization sessions, and viable tumors were detected with CBCT hepatic arteriography in about half the patients with elevating tumor markers and negative imaging studies.

Even though cTACE is regarded as a palliative treatment option for intermediate- or advanced-stage HCCs, a superselective cTACE is best suited to treat small nodular HCCs. Actually, cTACE is widely used for early-stage HCCs for the following reasons: $\left.{ }^{7} 1\right)$ Liver transplantation is limited by a shortage of donors, and candidates for surgical resection are often restricted by low liver volume, portal hypertension, old age, decreased performance status, and comorbidities; ${ }^{30}$ 2) A number of early-stage HCC patients are not good candidates for radiofrequency ablation (RFA) because of their challeng- ing anatomy or challenging tumor location; ${ }^{31}$ and 3 ) cTACE has a curative potential for small HCCs, as mentioned before. ${ }^{32}$ CBCT enables us to perform superselective chemoembolization for sub-centimeter-sized HCCs by demonstrating small occult lesions and their tumor feeders, which could not be demonstrated by DSA. ${ }^{33,34}$

\section{Detection of fine tumor-feeding arteries}

Out of the 228 tumor-feeding arteries of 142 tumors (1.99 $\pm 1.03 \mathrm{~cm}$ in diameter; range, $0.5-4.9 \mathrm{~cm}$ ) investigated in our study, 97.4\% could be detected and 95.2\% could be traced from the common hepatic artery to the tumor on the MPR and MIP images of CBCT. ${ }^{35}$ As a result, subsegmental superselective chemoembolization could be performed using CBCT hepatic arteriography without any additional angiography in $>90 \%$ of the tumors in $85 \%$ of the patients. In the recently published systemic review and meta-analysis, CBCT can significantly increase the detection of tumor-feeding arteries during chemoembolization. ${ }^{36}$

\section{3D roadmap and automated vessel tracking system}

Some studies have reported the technique of automated

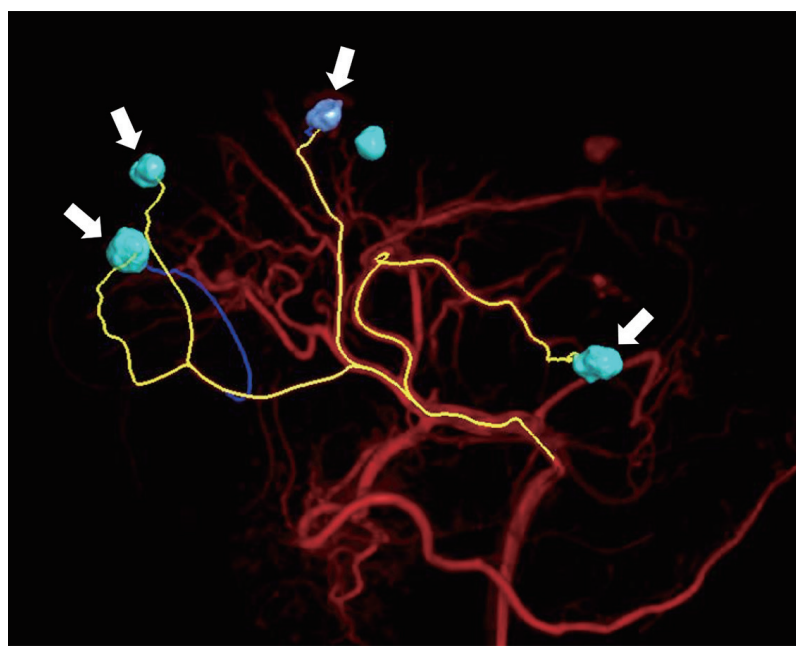

Figure 3. Depiction of the tumor-feeding arteries of four nodular tumors (arrows) using automated vessel tracking system (Emboguide; Phillips Medical Systems, Eindhoven, the Netherlands) in a 61-year-old woman. 

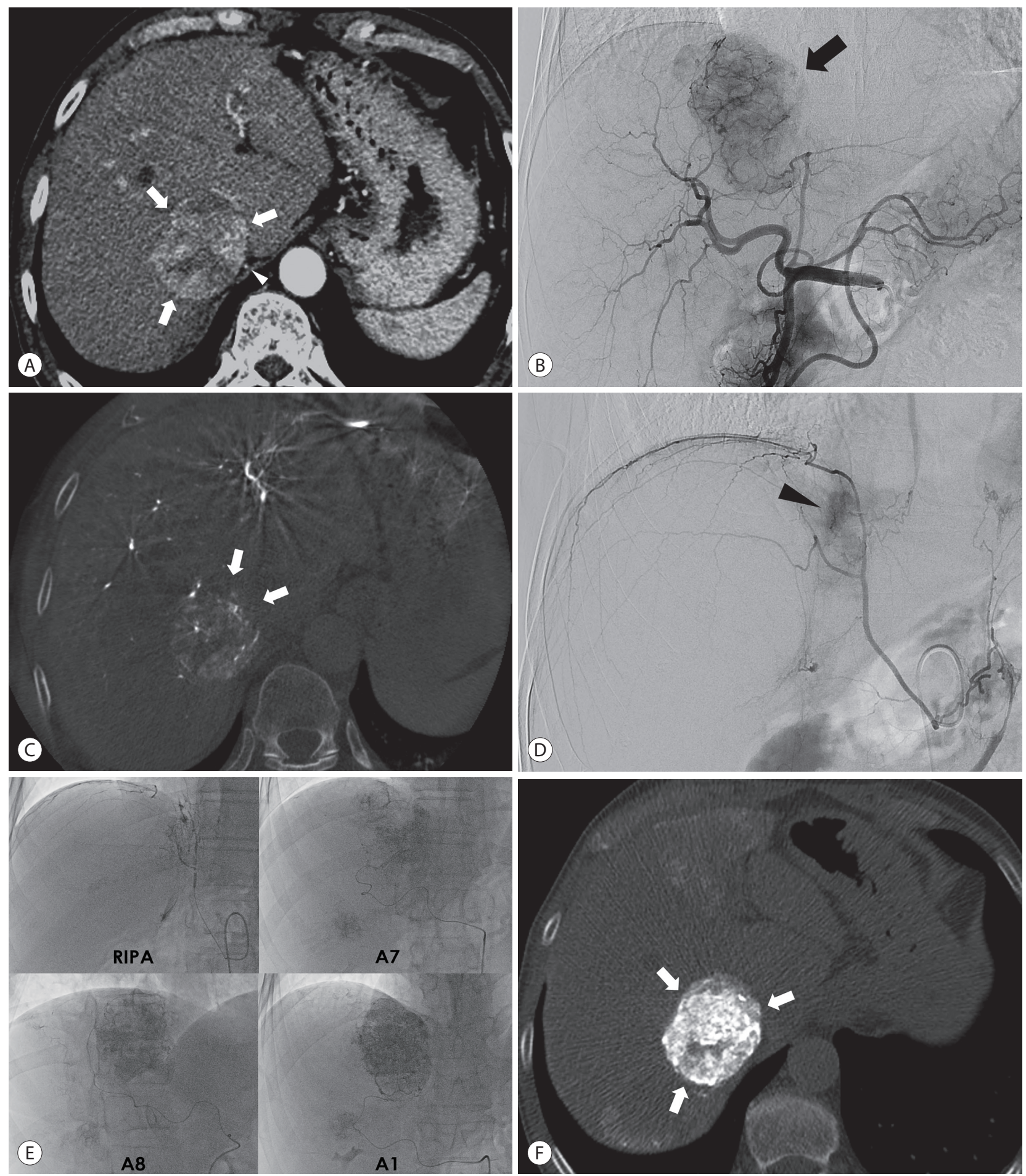

Figure 4. Recognition of extrahepatic collateral arterial supply to the tumor using CBCT images in a 62-year-old man. (A) Arterial phase image of CT shows large hypervascular tumor (arrows) in the paracaval portion of the liver. The RIPA is also demonstrated around the tumor (arrowhead). (B) Common hepatic angiography shows the hypervascular tumor (arrow). (C) Non-enhancing part (arrows) of the tumor is demonstrated in the axial image of CBCT but not in the preprocedural CT image (Fig. 4A), which suggests the presence of extrahepatic collateral artery supplying the tumor. (D) Angiography of the RIPA shows tumor staining. Angiography of the RIPA shows tumor staining (arrowhead). (E) Supserselective CTACE is performed through the RIPA, segment 7 hepatic artery, segment 8 hepatic artery, and caudate artery. (F) Immediate, unenhanced CT shows dense Lipiodol accumulation at the tumor (arrows). CBCT, cone-beam computed tomography; $\mathrm{CT}$, computed tomography; RIPA, right inferior phrenic artery; $\mathrm{CTACE}$, conventional transarterial chemoembolization. 
vessel tracking for detecting tumor feeders, ${ }^{37-39}$ which utilize 3 D visualizations (Fig. 3). Detectability rates of subsegmental tumor-feeders by the system are reported to be $81-93 \%$, which is higher than that by DSA. However, there is still a significant number of false-positive results. Therefore, automated vessel tracking system provides an objective second opinion, but the final decision concerning tumor-feeding arteries should be made by operators themselves after reviewing the MPR and MIP images. In addition to assisting tumor-feeder detection, automated vessel tracking and 3D roadmap function help us navigate the microcatheter to the target point through tortuous and branching arteries.

\section{Recognition of extrahepatic collateral blood supply}

At the initial presentation, HCCs with subcapsular location or exophytic growth might be receiving extrahepatic collateral supply. Extrahepatic collateral supply is more common in the bigger tumors. During repeated treatment sessions, the likelihood of extrahepatic supply for residual or recurrent tumor further increases. ${ }^{40}$ Therefore, the recognition
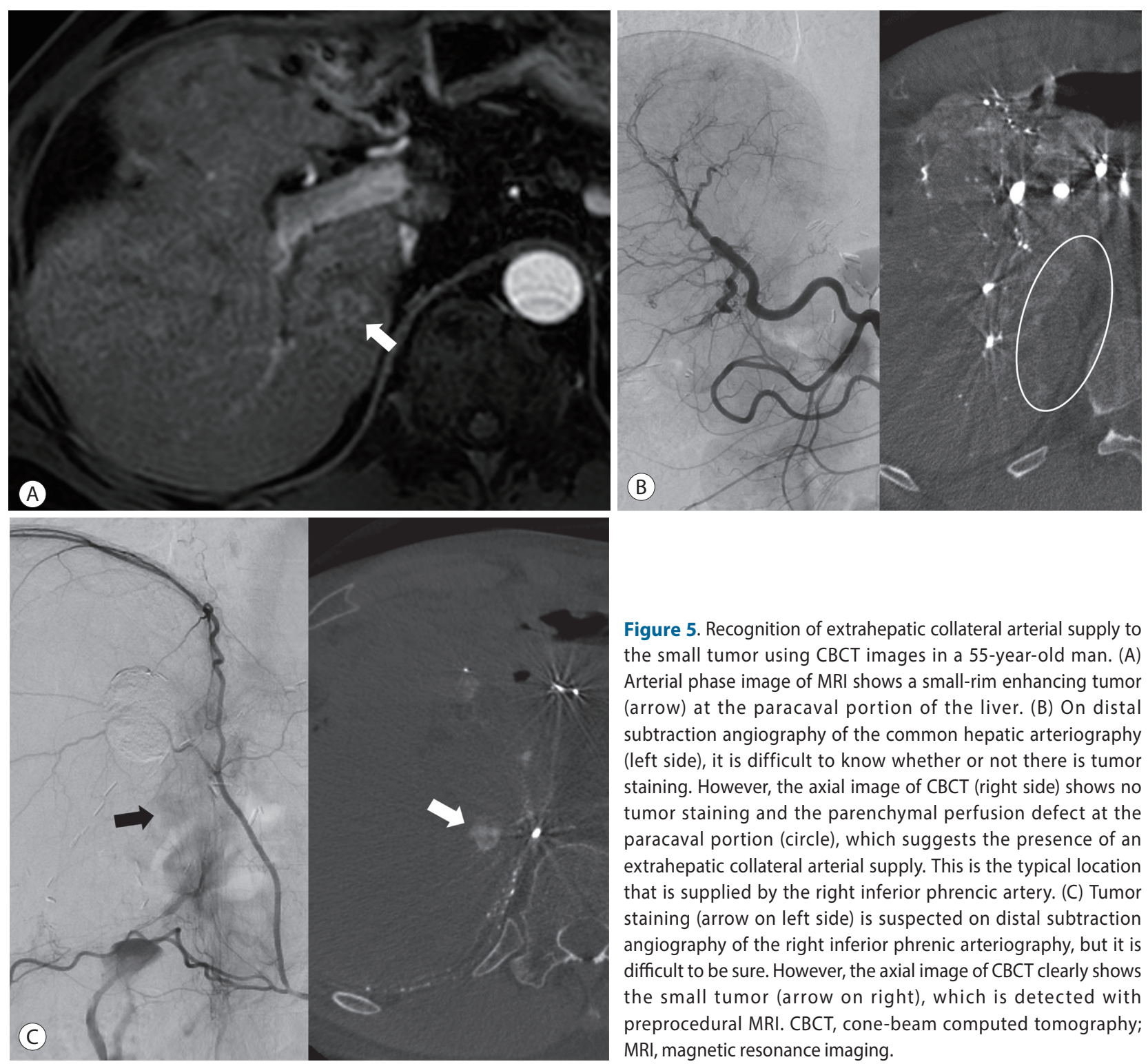

Figure 5. Recognition of extrahepatic collateral arterial supply to the small tumor using CBCT images in a 55-year-old man. (A) Arterial phase image of MRI shows a small-rim enhancing tumor (arrow) at the paracaval portion of the liver. (B) On distal subtraction angiography of the common hepatic arteriography (left side), it is difficult to know whether or not there is tumor staining. However, the axial image of CBCT (right side) shows no tumor staining and the parenchymal perfusion defect at the paracaval portion (circle), which suggests the presence of an extrahepatic collateral arterial supply. This is the typical location that is supplied by the right inferior phrencic artery. (C) Tumor staining (arrow on left side) is suspected on distal subtraction angiography of the right inferior phrenic arteriography, but it is difficult to be sure. However, the axial image of CBCT clearly shows the small tumor (arrow on right), which is detected with preprocedural MRI. CBCT, cone-beam computed tomography; $\mathrm{MRI}$, magnetic resonance imaging. 
of parasitic supply through extrahepatic collateral arteries is essential to achieve a successful outcome after chemoembolization. $^{41,42}$

It is not easy to detect the extrahepatic collateral supply to HCCs using conventional DSA, especially when a small HCC is totally supplied by an extrahepatic collateral artery. Large hypervascular tumor stain on hepatic angiography may mask the tumor area supplied by the extrahepatic collaterals because they are hidden behind. It is time consuming to perform selective angiography for all potential collateral arteries.

On CBCT hepatic arteriography, tomographic images clearly show the perfusion territory of hepatic arteries. If a tumor resides outside of the perfusion territory or shows a defect on hepatic artery perfusion, extrahepatic collateral arteries should be looked for (Figs. 4, 5). The CBCT scan of extrahepatic arteries directly demonstrates tumor stain and tumor feeding arteries from extrahepatic collaterals. In addition, it provides a comprehensive 3D roadmap of extrahepatic collateral arteries to avoid or minimize non-target embolization.

\section{Prevention of non-target embolization}

CBCT is highly recommended to improve the safety of chemoembolization by preventing or minimizing non-target embolization. Because CBCT hepatic arteriography provides more accurate information of the tumor location and tumorfeeding arteries, the area of liver damage can be minimized. Moreover, there are several non-hepatic arteries originating from the hepatic artery, including the cystic artery, falciform artery, accessory left gastric artery, right gastric artery, and left inferior phrenic artery. ${ }^{43}$ Skin injury, diaphragmatic injury, or gastrointestinal injury may develop as a result of nontarget embolization. CBCT provides accurate information on non-target vessels, and operators can avoid the embolization of non-target vessels.

\section{Immediate assessment of the completeness of chemoembolization}

It is possible to monitor the Lipiodol accumulation in tu- mor during chemoembolization with CBCT. Intraprocedural CBCT can clearly demonstrate the safety margin of chemoembolization around the tumor and the completeness of Lipiodol accumulation in the tumor. Based on the CBCT findings, operators can determine whether the tumor is completely treated or not and can decide the endpoint of the procedure. Miyayama et al. ${ }^{44}$ reported that intraprocedural monitoring using CBCT could decrease local tumor recurrence after the chemoembolization of small HCCs.

\section{Improvement of therapeutic efficacy of che- moembolization}

With these advantages of CBCT in chemoembolization, it is expected to improve local tumor control and overall survival rates of patients with HCC. Iwazawa et al. ${ }^{45}$ conducted a retrospective study comparing chemoembolization with and without $\mathrm{CBCT}$ assistance and reported significant improvement in the progression-free survival rates $(94 \%, 81 \%$, and $71 \%$ with CBCT, and $79 \%, 65 \%$, and $41 \%$ without, at 1-, 2-, and 3-years, respectively) and overall survival rates (43\%, $31 \%$, and $26 \%$ with CBCT, and $27 \%, 10 \%$, and $5 \%$ without, at 1-, 2-, and 3-years, respectively). Further prospective studies should be conducted to confirm the advantage of CBCTassisted chemoembolization with survival rates.

\section{RADIATION DOSE OF CBCT GUIDANCE IN CHEMOEMBOLIZATION}

The radiation dose of CBCT depends on the scan mode, angiography system, and manufacturer. According to our study using DynaCT (Siemens, Erlangen, Germany), the mean dose area product (DAP) and standard deviation (SD) of CBCT hepatic arteriography was $5,864.45 \pm 874.43 \mu \mathrm{Gy} \cdot \mathrm{m}^{2}$ (range, 2,055.4-7,787.3) and 204.7 $\pm 31.40 \mathrm{mGy}$ (range, 71.3304.0), respectively. ${ }^{25}$ Because of a higher radiation dose compared to single conventional hepatic arteriography, CBCT could increase stochastic risk (radiation-induced cancer). However, CBCT hepatic arteriography can provide enough information for superselective cTACE, eliminating the need for additional angiography. Kothary et al. ${ }^{46}$ demon- 
strated that $\mathrm{CBCT}$ can reduce the number of angiographic runs, thereby resulting in a negligible increase in dose-area product, which is considered as a surrogate for stochastic risks.

\section{CONCLUSION}

CBCT facilitates the detection of small occult HCCs and fine tumor-feeding arteries, recognition of extrahepatic collateral supply, navigation of a microcatheter to the target feeding arteries, prevention of non-target embolization, and intraprocedural assessment of the completeness of treatment with chemoembolization. These functions performed by CBCT ultimately improve the safety and efficacy of chemoembolization and may contribute to improving the prognosis of the patient with HCC.

\section{AUTHOR CONTRIBUTIONS}

I.J.L. and J.W.C. were responsible for the acquisition and interpretation of the data, and the drafting of the manuscript.

\section{Conflicts of Interest}

J.W.C. reported grants and lecture fees from Guerbet and BTG.

\section{REFERENCES}

1. Llovet JM, Real MI, Montaña X, Planas R, Coll S, Aponte J, et al. Arterial embolisation or chemoembolisation versus symptomatic treatment in patients with unresectable hepatocellular carcinoma: a randomised controlled trial. Lancet 2002;359:1734-1739.

2. Lo CM, Ngan H, Tso WK, Liu CL, Lam CM, Poon RT, et al. Randomized controlled trial of transarterial lipiodol chemoembolization for unresectable hepatocellular carcinoma. Hepatology 2002;35:11641171.

3. Korean Liver Cancer Study Group (KLCSG), National Cancer Center, Korea (NCC). 2014 Korean Liver Cancer Study Group-National Cancer Center Korea practice guideline for the management of hepatocellular carcinoma. Korean J Radiol 2015;16:465-522.

4. European Association for the Study of the Liver, European Association for the Study of the Liver. EASL clinical practice guidelines: management of hepatocellular carcinoma. J Hepatol 2018;69:182-
236.

5. Heimbach JK, Kulik LM, Finn RS, Sirlin CB, Abecassis MM, Roberts $L R$, et al. AASLD guidelines for the treatment of hepatocellular carcinoma. Hepatology 2018;67:358-380.

6. Yau T, Tang VY, Yao TJ, Fan ST, Lo CM, Poon RT. Development of Hong Kong Liver Cancer staging system with treatment stratification for patients with hepatocellular carcinoma. Gastroenterology 2014;146:1691-1700.e3.

7. Park JW, Chen M, Colombo M, Roberts LR, Schwartz M, Chen PJ, et al. Global patterns of hepatocellular carcinoma management from diagnosis to death: the BRIDGE Study. Liver Int 2015;35:21552166.

8. Golfieri R, Cappelli A, Cucchetti A, Piscaglia F, Carpenzano M, Peri $E$, et al. Efficacy of selective transarterial chemoembolization in inducing tumor necrosis in small $(<5 \mathrm{~cm})$ hepatocellular carcinomas. Hepatology 2011;53:1580-1589.

9. Golfieri R, Renzulli M, Mosconi C, Forlani L, Giampalma E, Piscaglia $F$, et al. Hepatocellular carcinoma responding to superselective transarterial chemoembolization: an issue of nodule dimension? J Vasc Interv Radiol 2013;24:509-517.

10. Takaki S, Sakaguchi H, Anai H, Tanaka T, Yamamoto K, Morimoto $K$, et al. Long-term outcome of transcatheter subsegmental and segmental arterial chemoemobolization using lipiodol for hepatocellular carcinoma. Cardiovasc Intervent Radiol 2012;35:544-554.

11. Gupta R, Grasruck M, Suess C, Bartling SH, Schmidt B, Stierstorfer $K$, et al. Ultra-high resolution flat-panel volume CT: fundamental principles, design architecture, and system characterization. Eur Radiol 2006;16:1191-1205.

12. Orth RC, Wallace MJ, Kuo MD; Technology Assessment Committee of the Society of Interventional R. C-arm cone-beam CT: general principles and technical considerations for use in interventional radiology. J Vasc Interv Radiol 2008;19:814-820.

13. Kim HC. Role of C-arm cone-beam CT in chemoembolization for hepatocellular carcinoma. Korean J Radiol 2015;16:114-124.

14. Matsui O, Kobayashi S, Sanada J, Kouda W, Ryu Y, Kozaka K, et al. Hepatocelluar nodules in liver cirrhosis: hemodynamic evaluation (angiography-assisted CT) with special reference to multi-step hepatocarcinogenesis. Abdom Imaging 2011;36:264-272.

15. Sasaki A, Kai S, Iwashita Y, Hirano S, Ohta M, Kitano S. Microsatellite distribution and indication for locoregional therapy in small hepatocellular carcinoma. Cancer 2005;103:299-306.

16. Kan Z, Ivancev K, Hägerstrand I, Chuang VP, Lunderquist A. In vivo microscopy of the liver after injection of Lipiodol into the hepatic artery and portal vein in the rat. Acta Radiol 1989;30:419-425.

17. Kan Z, Sato M, Ivancev $K$, Uchida B, Hedgpeth P, Lunderquist A, et al. Distribution and effect of iodized poppyseed oil in the liver after hepatic artery embolization: experimental study in several animal species. Radiology 1993;186:861-866. 
18. Kitao A, Zen Y, Matsui O, Gabata T, Nakanuma Y. Hepatocarcinogenesis: multistep changes of drainage vessels at CT during arterial portography and hepatic arteriography--radiologic-pathologic correlation. Radiology 2009;252:605-614.

19. Miyayama S, Matsui O, Yamashiro M, Ryu Y, Kaito K, Ozaki K, et al. Ultraselective transcatheter arterial chemoembolization with a $2-f$ tip microcatheter for small hepatocellular carcinomas: relationship between local tumor recurrence and visualization of the portal vein with iodized oil. J Vasc Interv Radiol 2007;18:365-376.

20. Iwamoto S, Yamaguchi T, Hongo O, Iwamoto H, Sanefuji H. Excellent outcomes with angiographic subsegmentectomy in the treatment of typical hepatocellular carcinoma: a retrospective study of local recurrence and long-term survival rates in 120 patients with hepatocellular carcinoma. Cancer 2010;116:393-399.

21. Kalender WA, Kyriakou Y. Flat-detector computed tomography (FD-CT). Eur Radiol 2007;17:2767-2779.

22. Virmani S, Ryu RK, Sato KT, Lewandowski RJ, Kulik L, Mulcahy $M F$, et al. Effect of C-arm angiographic CT on transcatheter arterial chemoembolization of liver tumors. J Vasc Interv Radiol 2007;18:1305-1309.

23. Iwazawa J, Ohue S, Mitani T, Abe H, Hashimoto N, Hamuro M, et al. Identifying feeding arteries during TACE of hepatic tumors: comparison of C-arm CT and digital subtraction angiography. AJR Am J Roentgenol 2009;192:1057-1063.

24. Meyer BC, Witschel M, Frericks BB, Voges M, Hopfenmüller W, Wolf $\mathrm{KJ}$, et al. The value of combined soft-tissue and vessel visualisation before transarterial chemoembolisation of the liver using Carm computed tomography. Eur Radiol 2009;19:2302-2309.

25. Lee IJ, Chung JW, Yin YH, Kim HC, Kim YI, Jae HJ, et al. Conebeam CT hepatic arteriography in chemoembolization for hepatocellular carcinoma: angiographic image quality and its determining factors. J Vasc Interv Radiol 2014;25:1369-1379; quiz 1379-.e1.

26. Tognolini A, Louie J, Hwang G, Hofmann L, Sze D, Kothary N. Carm computed tomography for hepatic interventions: a practical guide. J Vasc Interv Radiol 2010;21:1817-1823.

27. Meyer BC, Frericks BB, Voges M, Borchert M, Martus P, Justiz $J$, et al. Visualization of hypervascular liver lesions during TACE: comparison of angiographic C-arm CT and MDCT. AJR Am J Roentgenol 2008;190:W263-W269.

28. Iwazawa J, Ohue S, Hashimoto N, Abe H, Hamuro M, Mitani T. Detection of hepatocellular carcinoma: comparison of angiographic C-arm CT and MDCT. AJR Am J Roentgenol 2010;195:882-887.

29. Yu MH, Kim JH, Yoon JH, Kim HC, Chung JW, Han JK, et al. Role of C-arm CT for transcatheter arterial chemoembolization of hepatocellular carcinoma: diagnostic performance and predictive value for therapeutic response compared with gadoxetic acid-enhanced MRI. AJR Am J Roentgenol 2013;201:675-683.

30. Bruix J, Castells A, Bosch J, Feu F, Fuster J, Garcia-Pagan JC, et al.
Surgical resection of hepatocellular carcinoma in cirrhotic patients: prognostic value of preoperative portal pressure. Gastroenterology 1996;111:1018-1022.

31. Chen RX, Gan YH, Ge NL, Chen Y, Ma H, Wang Y, et al. Comparison of transarterial chemoembolization with radiofrequency ablation for unresectable Barcelona Clinic Liver Cancer stage 0/A hepatocellular carcinoma: a propensity score matching. J Gastroenterol Hepatol 2016;31:442-449.

32. Yang HJ, Lee JH, Lee DH, Yu SJ, Kim YJ, Yoon JH, et al. Small single-nodule hepatocellular carcinoma: comparison of transarterial chemoembolization, radiofrequency ablation, and hepatic resection by using inverse probability weighting. Radiology 2014;271:909-918.

33. Miyayama $S$, Yamashiro $M$, Okuda M, Yoshie $Y$, Sugimori N, Igarashi $S$, et al. Usefulness of cone-beam computed tomography during ultraselective transcatheter arterial chemoembolization for small hepatocellular carcinomas that cannot be demonstrated on angiography. Cardiovasc Intervent Radiol 2009;32:255-264.

34. Choi JW, Kim HC, Lee JH, Yu SJ, Cho EJ, Kim MU, et al. Cone beam CT-guided chemoembolization of probable hepatocellular carcinomas smaller than $1 \mathrm{~cm}$ in patients at high risk of hepatocellular carcinoma. J Vasc Interv Radiol 2017;28:795-803.e1.

35. Lee IJ, Chung JW, Yin YH, Kim HC, Kim YI, Jae HJ, et al. Conebeam computed tomography (CBCT) hepatic arteriography in chemoembolization for hepatocellular carcinoma: performance depicting tumors and tumor feeders. Cardiovasc Intervent Radiol 2015;38:1218-1230.

36. Pung L, Ahmad M, Mueller K, Rosenberg J, Stave C, Hwang GL, et al. The role of cone-beam CT in transcatheter arterial chemoembolization for hepatocellular carcinoma: a systematic review and meta-analysis. J Vasc Interv Radiol 2017;28:334-341.

37. Deschamps F, Solomon SB, Thornton RH, Rao P, Hakime A, Kuoch $V$, et al. Computed analysis of three-dimensional cone-beam computed tomography angiography for determination of tumorfeeding vessels during chemoembolization of liver tumor: a pilot study. Cardiovasc Intervent Radiol 2010;33:1235-1242.

38. Iwazawa J, Ohue S, Hashimoto N, Muramoto O, Mitani T. Clinical utility and limitations of tumor-feeder detection software for liver cancer embolization. Eur J Radiol 2013;82:1665-1671.

39. Miyayama S, Yamashiro M, Hashimoto M, Hashimoto N, Ikuno M, Okumura $\mathrm{K}$, et al. Identification of small hepatocellular carcinoma and tumor-feeding branches with cone-beam CT guidance technology during transcatheter arterial chemoembolization. J Vasc Interv Radiol 2013;24:501-508.

40. Chung JW, Kim HC, Yoon JH, Lee HS, Jae HJ, Lee W, et al. Transcatheter arterial chemoembolization of hepatocellular carcinoma: prevalence and causative factors of extrahepatic collateral arteries in 479 patients. Korean J Radiol 2006;7:257-266. 
41. Kim HC, Chung JW, Lee W, Jae HJ, Park JH. Recognizing extrahepatic collateral vessels that supply hepatocellular carcinoma to avoid complications of transcatheter arterial chemoembolization. Radiographics 2005;25 Suppl 1:S25-S39.

42. Moustafa AS, Abdel Aal AK, Ertel N, Saad N, DuBay D, Saddekni S. Chemoembolization of hepatocellular carcinoma with extrahepatic collateral blood supply: anatomic and technical considerations. Radiographics 2017;37:963-977.

43. Song SY, Chung JW, Lim HG, Park JH. Nonhepatic arteries originating from the hepatic arteries: angiographic analysis in 250 patients. J Vasc Interv Radiol 2006;17:461-469.

44. Miyayama S, Yamashiro M, Hashimoto M, Hashimoto N, Ikuno M, Okumura $\mathrm{K}$, et al. Comparison of local control in transcatheter ar- terial chemoembolization of hepatocellular carcinoma $\leq 6 \mathrm{~cm}$ with or without intraprocedural monitoring of the embolized area using cone-beam computed tomography. Cardiovasc Intervent Radiol 2014;37:388-395.

45. Imazawa J, Ohue S, Hashimoto N, Muramoto O, Mitani T. Survival after $\mathrm{C}$-arm CT-assisted chemoembolization of unresectable hepatocellular carcinoma. Eur J Radiol 2012;81:3985-3992.

46. Kothary N, Abdelmaksoud MH, Tognolini A, Fahrig R, Rosenberg J, Hovsepian DM, et al. Imaging guidance with C-arm CT: prospective evaluation of its impact on patient radiation exposure during transhepatic arterial chemoembolization. J Vasc Interv Radiol 2011;22:1535-1543. 